Pacific Journal of Mathematics

ON THE LERCH ZETA FUNCTION 


\title{
ON THE LERCH ZETA FUNCTION
}

\author{
T. M. Apostol
}

1. Introduction. The function $\phi(x, a, s)$, defined for $R s>1, x$ real, $a \neq$ negative integer or zero, by the series

$$
\phi(x, a, s)=\sum_{n=0}^{\infty} \frac{e^{2 n \pi i x}}{(a+n)^{s}},
$$

was investigated by Lipschitz $[4 ; 5]$, and Lerch [3]. By use of the classic method of Riemann, $\phi(x, a, s)$ can be extended to the whole $s$-plane by means of the contour integral

$$
I(x, a, s)=\frac{1}{2 \pi i} \int_{C} \frac{z^{s-1} e^{a z}}{1-e^{z+2 \pi i x}} d z
$$

where the path $C$ is a loop which begins at $-\infty$, encircles the origin once in the positive direction, and returns to $-\infty$. Since $I(x, a, s)$ is an entire function of $s$, and we have

$$
\phi(x, a, s)=\Gamma(1-s) I(x, a, s),
$$

this equation provides the analytic continuation of $\phi$. For integer values of $x$, $\phi(x, a, s)$ is a meromorphic function (the Hurwitz zeta function) with only a simple pole at $s=1$. For nonintegral $x$ it becomes an entire function of $s$. For $0<x<1$, $0<a<1$, we have the functional equation

$$
\begin{aligned}
& \phi(x, a, 1-s) \\
& \quad=\frac{\Gamma(s)}{(2 \pi)^{s}}\left\{e^{\pi i(s / 2-2 a x)} \phi(-a, x, s)+e^{\pi i(-s / 2+2 a(1-x))} \phi(a, 1-x, s)\right\},
\end{aligned}
$$

first given by Lerch, whose proof follows the lines of the first Riemann proof of the functional equation for $\zeta(s)$ and uses Cauchy's theorem in connection with the contour integral (1.2). 
In the present paper, $\$ 2$ contains a proof of (1.4) based on the transformation theory of theta-functions. This proof is of particular interest because the usual approach (Riemann's second method) does not lead to the functional equation (1.4) as might be expected but to a different functional relationship (equation (2.4) below). Further properties of $\phi(x, a, s)$, having no analogue in the case of $\zeta(s)$, are needed to carry this method through to obtain (1.4).

In $\S 3$ we evaluate the function $\phi(x, a, s)$ for negative integer values of $s$. These results are expressible in closed form by means of a sequence of functions $\beta_{n}\left(a, e^{2 \pi i x}\right)$ which are polynomials in $a$ and rational functions in $e^{2 \pi i x}$. These functions are closely related to Bernoulli polynomials; their basic properties also are developed here.

2. Functional Equation for $\phi(x, a, s)$. The theta-function

$$
\vartheta_{3}(y \mid \tau)=\sum_{n=-\infty}^{\infty} \exp \left(\pi i n^{2} \tau+2 i n y\right)
$$

has the transformation formula $[6, p .475]$

$$
\vartheta_{3}(y \mid \tau)=(-i \tau)^{-1 / 2} \exp \left(\frac{y^{2}}{\pi i \tau}\right) \vartheta_{3}\left(\frac{y}{\tau} \mid \frac{-1}{\tau}\right) .
$$

If we let

$$
\theta(x, a, z)=\exp \left(-\pi a^{2} z\right) \vartheta_{3}(\pi x+\pi i a z \mid i z)=\sum_{n=-\infty}^{\infty} \exp \left(2 n \pi i x-\pi z(a+n)^{2}\right),
$$

then we have the functional equation

$$
\theta(a,-x, 1 / z)=[\exp (2 \pi i a x)] z^{1 / 2} \theta(x, a, z) .
$$

The key to Riemann's second method is the formal identity

$$
\pi^{-s / 2} \Gamma(s / 2) \sum_{n=1}^{\infty} a_{n} f_{n}^{-s / 2}=\int_{0}^{\infty} z^{s / 2-1} \sum_{n=1}^{\infty} a_{n} \exp \left(-\pi z f_{n}\right) d z
$$

Taking first $a_{n}=\exp [(2 \pi i(n-1) x)], f_{n}=(n-1+a)^{2}$ in (2.2), and then $a_{n}=\exp (-2 \pi i n x), f_{n}=(n-a)^{2}$, we obtain 


$$
\begin{aligned}
\pi^{-s / 2} \Gamma(s / 2)\{\phi(x, a, s)+ & \exp (-2 \pi i x) \phi(-x, 1-a, s)\} \\
& =\left(\int_{1}^{\infty}+\int_{0}^{1}\right) z^{s / 2-1} \theta(x, a, z) d z
\end{aligned}
$$

In the second integral in (2.3) we apply (2.1) and replace $z$ by $1 / z$. Denoting the expression in braces by $\Lambda(x, a, s)$, replacing $s$ by $1-s, x$ by $-a, a$ by $x$, using $\theta(-a, x, z)=\theta(a,-x, z)$, and the relation

$$
\pi^{1 / 2-s} \Gamma(s / 2) / \Gamma\left(\frac{1-s}{2}\right)=2(2 \pi)^{-s} \cos (\pi s / 2) \Gamma(s),
$$

we are led to

(2.4) $\Lambda(x, a, 1-s)=2(2 \pi)^{-s} \cos (\pi s / 2) \Gamma(s) \exp (-2 \pi i a x) \Lambda(-a, x, s)$.

Thus Riemann's method gives us a functional equation for $\Lambda$ instead of (1.4). At this point we introduce the differential-difference equations satisfied by $\phi$, namely:

$$
\frac{\partial \phi(x, a, s)}{\partial a}=-s \phi(x, a, s+1)
$$

and

$$
\frac{\partial \phi(x, a, s)}{\partial x}+2 \pi i a \phi(x, a, s)=2 \pi i \phi(x, a, s-1) .
$$

The first of these follows at once from (1.1). To obtain (2.6) we first write

$$
\phi(x, a, s)=\exp (-2 \pi i a x) \sum_{n=0}^{\infty} \frac{\exp [2 \pi i(n+a) x]}{(n+a)^{s}}
$$

before differentiating with respect to $x$. The equations hold for all $s$ by analytic continuation.

The proof of (1.4) as a consequence of (2.4) now proceeds as follows. We differentiate both sides of (2.4) with respect to the variable $a$, using (2.5) on the left and (2.6) on the right, and replace $s$ by $s+1$ in the resulting equation. This 
leads to the relation

$$
\begin{aligned}
\phi(x, a, 1-s)-\exp (-2 \pi i x) \phi(-x, 1-a, 1-s) \\
=2 i(2 \pi)^{-s} \sin (\pi s / 2) \Gamma(s) \\
\quad \quad \times[\exp (-2 \pi i a x) \phi(-a, x, s)-\exp (-2 \pi i a(1-x)) \phi(a, 1-x, s)] .
\end{aligned}
$$

Adding this equation to (2.4) gives the desired relation (1.4).

This method has already been used by N. J. Fine [1] to derive the functional equation of the Hurwitz zeta function. Fine's proof uses (2.5) with $x=0$. In our proof of (1.4) it is essential that $x \neq 0$ since we have occasion to interchange the variables $x$ and $a$, and $\phi(x, a, s)$ is not regular for $a=0$; hence Fine's proof is not a special case of ours. Furthermore, putting $x=0$ in (1.4) does not yield the Hurwitz functional equation, although this can be obtained from (1.4) as shown elsewhere by the author.

3. Evaluation of $\phi(x, a,-n)$. If $x$ is an integer, then $\phi(x, a, s)$ reduces to the Hurwitz zeta function $\zeta(s, a)$ whose properties are well known [6, pp. 265-279] . For nonintegral $x$ the analytic character of $\phi$ is quite different from that of $\zeta(s, a)$, and in what follows we assume that $x$ is not an integer.

The relation (2.6) can be used to compute recursively the values of $\phi(x, a, s)$ for $s=-1,-2,-3, \cdots$ As a starting point we compute the value at $s=0$ by substituting in (1.2). The value of the integral reduces to the residue of the integrand at $z=0$ and gives us

$$
\phi(x, a, 0)=\frac{1}{1-\exp (2 \pi i x)}=(i / 2) \cot \pi x+1 / 2 .
$$

Using (2.6) we obtain

$\phi(x, a,-1)=(a / 2)(i \cot \pi x+1)-(1 / 4) \csc ^{2} \pi x$,

$\phi(x, a,-2)=\left(a^{2} / 2\right)(i \cot \pi x+1 / 4)-(a / 2) \csc ^{2} \pi x-(i / 4) \cot \pi x \csc ^{2} \pi x$.

If we put $s=-n$ in (1.2) and use Cauchy's residue theorem we obtain, for $n \geq 0$, the relation

$$
\phi(x, a,-n)=-\frac{\beta_{n+1}\left(a, e^{2 \pi i x}\right)}{n+1},
$$


where $\beta_{n}(a, \alpha)$ is defined by the generating function

$$
z \frac{e^{a z}}{\alpha e^{z}-1}=\sum_{n=0}^{\infty} \frac{\beta_{n}(a, \alpha)}{n !} z^{n} .
$$

When $\alpha=1, \beta_{n}(a, \alpha)$ is the Bernoulli polynomial $B_{n}(a)$. For our purposes we assume $\alpha \neq 1$, and in the remainder of this section we give the main properties of the functions $\beta_{n}(a, \alpha)$.

Writing $\beta_{n}(\alpha)$ instead of $\beta_{n}(0, \alpha)$ we obtain from (3.1):

$$
\beta_{n}(a, a)=\sum_{k=0}^{n}\left(\begin{array}{l}
n \\
k
\end{array}\right) \beta_{k}(a) a^{n-k}
$$

from which we see that the functions $\beta_{n}(a, \alpha)$ are polynomials in the variable $a$. The defining equation (3.1) also leads to the difference equation

$$
\alpha \beta_{n}(a+1, \alpha)-\beta_{n}(a, \alpha)=n a^{n-1} \quad(n \geq 1) .
$$

Taking $a=0$ we obtain, for $n=1$, the relation

$$
a \beta_{1}(1, \alpha)=1+\beta_{1}(a)
$$

while for $n \geq 2$ we have

$$
\alpha \beta_{n}(1, \alpha)=\beta_{n}(\alpha) .
$$

Putting $a=1$ in (3.2) now allows us to compute the functions $\beta_{n}(\alpha)$ recursively by means of

$$
\beta_{n}(1, \alpha)=\sum_{k=0}^{n}\left(\begin{array}{l}
n \\
k
\end{array}\right) \beta_{k}(a)
$$

and (3.4), (3.5). From (3.1) we obtain $\beta_{0}(\alpha)=0$; the next few functions are found to be :

$$
\begin{aligned}
& \beta_{1}(\alpha)=\frac{1}{a-1}, \quad \beta_{2}(a)=-\frac{2 \alpha}{(\alpha-1)^{2}}, \quad \beta_{3}(a)=\frac{3 \alpha(\alpha+1)}{(a-1)^{3}}, \\
& \beta_{4}(a)=-\frac{4 \alpha\left(\alpha^{2}+4 \alpha+1\right)}{(\alpha-1)^{4}}, \quad \beta_{5}(\alpha)=\frac{5 \alpha\left(\alpha^{3}+11 \alpha^{2}+11 \alpha+1\right)}{(\alpha-1)^{5}},
\end{aligned}
$$




$$
\beta_{6}(\alpha)=-\frac{6 \alpha\left(\alpha^{4}+26 \alpha^{3}+66 \alpha^{2}+26 \alpha+1\right)}{(\alpha-1)^{6}} .
$$

The general formula is

$$
\beta_{n}(\alpha)=\frac{n \alpha}{(\alpha-1)^{n}} \sum_{s=1}^{n-1}(-1)^{s} s ! \alpha^{s-1}(\alpha-1)^{n-1-s} \oint_{n-1}^{(s)}
$$

where $\oiint_{k}^{(j)}$ are Stirling numbers of the second kind defined by

$$
\oiint_{k}^{(j)}=\frac{\triangle^{j} 0^{k}}{j !},
$$

with

$$
\Delta^{j} 0^{n}=\left(\Delta^{j} x^{n}\right)_{x=0}, \quad \Delta^{j} 0^{n}=0 \text { if } j>n, \quad \Delta^{0} 0^{0}=1,
$$

in the usual notation of finite differences. (A short table of Stirling numbers is given in [2].)

To prove (3.7) we put

$$
g(z, \alpha)=\frac{1}{\alpha e^{z}-1}=\frac{1}{\alpha-1}\left(1+\sum_{n=1}^{\infty}\left(\frac{\alpha}{1-\alpha}\right)^{n}\left(e^{z}-1\right)^{n}\right) .
$$

Using Herschel's theorem $\left[2\right.$, p. 73] which expresses $\left(e^{z}-1\right)^{n}$ as a power series in $z$ we obtain

$$
(\alpha-1) g(z, \alpha)=1+\sum_{m=1}^{\infty} \sum_{s=1}^{m}\left(\frac{\alpha}{1-\alpha}\right)^{s} \frac{s !}{m !} \oiint_{m}^{(s)} z^{m} .
$$

Comparing with

$$
g(z, \alpha)=\sum_{0}^{\infty} \beta_{n}(\alpha) \frac{z^{n}}{n !}
$$

we get (3.7).

The following further properties of the numbers $\beta_{n}(a, \alpha)$, which closely resemble well-known formulas for Bernoulli polynomials, are easy consequences of 
the above :

$$
\begin{aligned}
\frac{\partial^{p}}{\partial a^{p}} \beta_{n}(a, \alpha) & =\frac{n !}{(n-p) !} \beta_{n-p}(a, \alpha) & (0 \leq p \leq n), \\
\beta_{n}(a+b, \alpha) & =\sum_{k=0}^{n}\left(\begin{array}{l}
n \\
k
\end{array}\right) \beta_{k}(a, \alpha) b^{n-k}, & \\
\int_{a}^{b} \beta_{n}(t, \alpha) d t & =\frac{\beta_{n+1}(b, \alpha)-\beta_{n+1}(a, \alpha)}{n+1} & (n \geq 0) .
\end{aligned}
$$

Taking $a=b-1$ and using (3.3), we can also use this last equation to obtain the functions $\beta_{n}(a, \alpha)$ recursively by successive integration of polynomials.

As a final result, taking $a=0,1,2, \cdots, m-1$ in (3.3) and summing we obtain

$$
\sum_{a=0}^{m-1} a^{n}=\frac{\alpha-1}{n+1} \sum_{a=1}^{m} \beta_{n+1}(a, \alpha)+\frac{\beta_{n+1}(m, \alpha)-\beta_{n+1}(\alpha)}{n+1},
$$

a generalization of the famous formula giving $\Sigma a^{n}$ in terms of Bernoulli polynomials. This result is somewhat surprising because of the appearance of the parameter $\alpha$ on the right. (When $\alpha=1$, (3.8) reduces to the Bernoulli formula.)

\section{REFERENCES}

1. N. J. Fine, Note on the Hurwitz zeta-Function, Proc. Amer. Math. Soc., scheduled to appear in vol. 2 (1951).

2. T. Fort, Finite Differences, Clarendon Press, Oxford, 1948. 19-24.

3. M. Lerch, Note sur la fonction $\mathcal{R}(w, x, s)=\sum_{k=0}^{\infty} \frac{e^{2 k \pi i x}}{(w+k)^{s}}$, Acta Math. 11 (1887),

4. R. Lipschitz, Untersuchung einer aus vier Elementen gebildeten Reihe, J. Reine Angew. Math. 54 (1857), 313-328.

5.

Untersuchung der Eigenschaften einer Gattung von unendlichen Reihen, J. Reine Angew. Math. 105 (1889), 127-156.

6. E. T. Whittaker and G. N. Watson, Modern Analysis, University Press, Cambridge, England, 1945.

California Institute of TeGhnology 



\title{
PACIFIC JOURNAL OF MATHEMATICS
}

\section{EDITORS}

\author{
Herbert BuSEMANN \\ R. M. RoBINSON \\ University of Southern California \\ University of California \\ Los Angeles 7, California \\ Berkeley 4, California \\ E. F. BEC KENBACH, Managing Editor \\ University of California \\ Los Angeles 24, California
}

\section{ASSOCIATE EDITORS}
R. P. DILWORTH
P. R. HALMOS
BØRGE JESSEN
J. J. STOKER
HERBERT FEDERER
HEINZ HOPF
PAUL LÉVY
MARSHALL HALL
R. D. JAMES
GEORGE PÓLYA
E. G. STRAUS
KÖSAKU YOSIDA

\section{SPONSORS}

UNIVERSITY OF BRITISH COLUMBIA

CALIFORNIA INSTITUTE OF TECHNOLOGY

UNIVERSITY OF CALIFORNIA, BERKELEY

UNIVERSITY OF CALIFORNIA, DAVIS

UNIVERSITY OF CALIFORNIA, LOS ANGELES

UNIVERSITY OF CALIFORNIA, SANTA BARBARA

OREGON STATE COLLEGE

UNIVERSITY OF OREGON
UNIVERSITY OF SOUTHERN CALIFORNIA

STANFORD UNIVERSITY

WASHINGTON STATE COLLEGE

UNIVERSITY OF WASHINGTON

AMERICAN MATHEMATICAL SOCIETY

NATIONAL BUREAU OF STANDARDS, INSTITUTE FOR NUMERIGAL ANALYSIS

Mathematical papers intended for publication in the Pacific Journal of Mathematics should be typewritten (double spaced), and the author should keep a complete copy. Manuscripts may be sent to any of the editors. All other communications to the editors should be addressed to the managing editor, E. F. Beckenbach, at the address given above.

Authors are entitled to receive 100 free reprints of their published papers and may obtain additional copies at cost.

The Pacific Journal of Mathematics is published quarterly, in March, June, September, and December. The price per volume (4 numbers) is $\$ 8.00$; single issues, $\$ 2.50$. Spécial price to individual faculty members of supporting institutions and to members of the American Mathematical Society: $\$ 4.00$ per volume; single issues, $\$ 1.25$.

Subscriptions, orders for back numbers, and changes of address should be sent to the publishers, University of California Press, Berkeley 4, California.

UNIVERSITY OF CALIFORNIA PRESS - BERKELEY AND LOS ANGELES 


\section{Pacific Journal of Mathematics}

\section{Vol. 1, No. $2 \quad$ December, 1951}

Tom M. (Mike) Apostol, On the Lerch zeta function ................. 161

Ross A. Beaumont and Herbert S. Zuckerman, A characterization of the subgroups of the additive rationals ....................... 169

Richard Bellman and Theodore Edward Harris, Recurrence times for the Ehrenfest model................................... 179

Stephen P.L. Diliberto and Ernst Gabor Straus, On the approximation of a function of several variables by the sum of functions of fewer

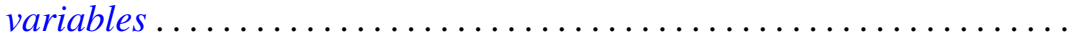

Isidore Isaac Hirschman, Jr. and D. V. Widder, Convolution transforms with complex kernels ................................ 211

Irving Kaplansky, A theorem on rings of operators .............. 227

W. Karush, An iterative method for finding characteristic vectors of a symmetric matrix............................... 233

Henry B. Mann, On the number of integers in the sum of two sets of positive integers ......................................... 249

William H. Mills, A theorem on the representation theory of Jordan

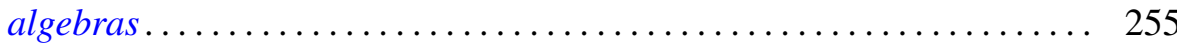

Tibor Radó, An approach to singular homology theory.............. 265

Otto Szász, On some trigonometric transforms ................... 291

James G. Wendel, On isometric isomorphism of group algebras ......... 305

George Milton Wing, On the $L^{p}$ theory of Hankel transforms ... 